\title{
A near-infrared variability campaign of TMR-1: New light on the nature of the candidate protoplanet TMR-1C ${ }^{\star}$
}

\author{
B. Riaz ${ }^{1}$, E. L. Martín ${ }^{2}$, M. G. Petr-Gotzens ${ }^{3}$, and J.-L. Monin ${ }^{4}$ \\ ${ }^{1}$ Centre for Astrophysics Research, Science \& Technology Research Institute, University of Hertfordshire, Hatfield, AL10 9AB, UK \\ e-mail: b.riaz@herts.ac.uk \\ 2 Centro de Astrobiología (CSIC/INTA), 28850 Torrejón de Ardoz, Madrid, Spain \\ ${ }^{3}$ European Southern Observatory, Karl-Schwarzschild-Str. 2, 85748 Garching bei München, Germany \\ ${ }^{4}$ UJF-Grenoble/CNRS-INSU, Institut de Planétologie et d'Astrophysique de Grenoble (IPAG) UMR 5274, 38041 Grenoble, France
}

Received 22 April 2013 / Accepted 18 June 2013

\begin{abstract}
We present a near-infrared (NIR) photometric variability study of the TMR-1 system, a Class I protobinary located in the Taurus molecular cloud. Our aim is to confirm NIR variability for the candidate protoplanet, TMR-1C, located at a separation of about $10^{\prime \prime}$ ( $1000 \mathrm{AU})$ from the protobinary. We conducted a photometric monitoring campaign between October 2011 and January 2012 using the CFHT/WIRCam imager. We were able to obtain 44 epochs of observations in each of the $H$ and $K_{\mathrm{s}}$ filters, resulting in high-quality photometry with uncertainties of less than one-tenth of a magnitude. The shortest time difference between two epochs is $\sim 14$ min, and the longest is $\sim 4$ months. Based on the final accuracy of our observations, we do not find any strong evidence of short-term NIR variability at amplitudes of $\geq 0.15-0.2 \mathrm{mag}$ for TMR-1C or TMR-1AB. Our present observations, however, have reconfirmed the large-amplitude long-term variations in the NIR emission for TMR-1C, which were observed between 1998 and 2002, and have also shown that no particular correlation exists between the brightness and the color changes. The object TMR-1C became brighter in the $H$ band by $\sim 1.8$ mag between 1998 and 2002, and then fainter again by $\sim 0.7$ mag between 2002 and 2011. In contrast, TMR-1C became continually brighter in the $K_{\mathrm{s}}$ band in the period between 1998 and 2011 . The $\left(H-K_{\mathrm{s}}\right)$ color for TMR-1C shows large variations, from a red value of $1.3 \pm 0.07$ and $1.6 \pm 0.05 \mathrm{mag}$ in 1998 and 2000, to a much bluer color of $-0.1 \pm 0.5 \mathrm{mag}$ in 2002 , and then again a red color of $1.1 \pm 0.08 \mathrm{mag}$ in 2011. The difference in the variability trends observed in the $H$ and $K_{\mathrm{s}}$ bands suggests the presence of more than one origin for the observed variations. The observed variability from 1998 to 2011 suggests that TMR-1C becomes fainter when it gets redder, as expected from variable extinction, while the brightening observed in the $K_{\mathrm{s}}$ band could be due to physical variations in the inner disk structure of TMR-1C.

We have argued in favor of TMR-1C being a young stellar object (YSO), rather than a faint background star passing behind some foreground material. There may exist short-term NIR variations at an amplitude level lower than our detection limit $(\sim 0.2 \mathrm{mag})$, which would be consistent with the YSO hypothesis. If the observed long-term variability is due to foreground extinction, then we would expect simultaneous brightening/dimming in the $H$ and $K_{\mathrm{s}}$ bands, which we do not find to be the case. Variable foreground extinction is also expected to occur over a large spatial scale; we monitored several other objects within $4^{\prime} \times 4^{\prime}$ of the TMR-1 system, and found only two objects which show long-term variations, indicating that this is not a large-scale effect. The NIR colors for TMR-1C obtained using the high-precision photometry from 1998, 2000, and 2011 observations are similar to the protostars in Taurus, suggesting that it could be a faint dusty Class I source. This object is thus a strong candidate YSO, but final confirmation as a protoplanet remains elusive and requires further investigation.

Our study has also revealed two new variable sources in the vicinity of TMR-1AB that show long-term variations of $\sim 1-2$ mag in the NIR colors between 2002 and 2011. The proper motions measured for TMR-1AB and TMR-1C are $-40,+58$ mas/yr and $-22,+5$ mas/yr, respectively, with an uncertainty of $\sim 31$ mas/yr. A larger baseline of 20 years or more is required to confidently confirm the physical association of TMR-1AB and $\mathrm{C}$.
\end{abstract}

Key words. stars: individual: TMR-1 - stars: protostars - planetary systems - stars: variables: T Tauri, Herbig Ae/Be stars: pre-main sequence - stars: individual: TMR-1C

\section{Introduction}

A notable characteristic of young pre-main sequence stars is photometric variability. Optical and near-infrared (NIR) monitoring observations can probe temperature, opacity, and geometrical variations in the circumstellar environment of a young star. Photometric variability studies conducted in several clusters have shown that young stellar objects (YSOs) exhibit both periodic and aperiodic variations, with amplitudes of $0.1-1$ mag and timescales of minutes to years (e.g.,

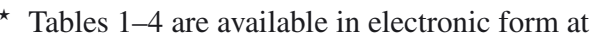
http://www. aanda.org
Bouvier et al. 1993; Herbst et al. 1994; Kenyon \& Hartmann 1995; Carpenter et al. 2001). The periodic variations yield information on stellar rotational periods and the sizes and temperatures of cool magnetic or hot accretion spots on the stellar photosphere (e.g., Bouvier et al. 1993). Aperiodic variability may arise from certain temporally changing inhomogeneities in the circumstellar environment, for example, or the ambient molecular cloud, or because of an irregular mass accretion rate (e.g., Herbst et al. 1994; Carpenter et al. 2001).

The star TMR-1 (IRAS 04361+2547) is a deeply embedded Class I protostellar system located in the Taurus molecular cloud. Near-infrared imaging and millimeter interferometry 
show a bipolar outflow extending southeast to the northwest (Terebey et al. 1990). This protostar has an estimated stellar mass of $\sim 0.5 M_{\odot}$, a bolometric luminosity of $\sim 3 L_{\odot}$, and an $A_{V}$ of $28 \pm 2$ mag (Terebey et al. 1990). Terebey et al. (1998; hereafter T98) presented Hubble Space Telescope (HST)/NICMOS observations of TMR-1, and were able to resolve this protostar into two point sources, A and B (the northern component was named A), at a separation of $0.31^{\prime \prime}$. A third, fainter, point source, TMR-1C, was detected at a separation of about $10^{\prime \prime}$ ( 1000 AU) from the protobinary. Assuming that TMR-1C is located in Taurus and is at the same age as TMR-1AB, T98 estimated a bolometric luminosity of approximately $10^{-3} L_{\odot}$ and a mass of $2-5 M_{\mathrm{J}}$ for this source, thus classifying it as a candidate protoplanet. Their observations also revealed a narrow filamentlike structure extending southeast of the central proto-binary system towards TMR-1C and led them to suggest a morphology in which the candidate protoplanet may have been ejected from the TMR-1 system, with the filament traversing the ejection path. However, follow-up low-resolution $(R \sim 120)$ NIR spectroscopy obtained by Terebey et al. (2000) with the Keck/NIRC instrument showed a featureless spectrum, and it was suggested that this may be a background star.

Two recently published studies by Riaz \& Martín (2011) and Petr-Gotzens et al. (2010) strongly argued that TMR-1C is most likely a YSO, possibly surrounded by a highly inclined disk, and not an extincted background star. In Riaz \& Martín (2011), we reported large-amplitude variations of $\sim 1-2$ mag for TMR-1C in the $H$ and $K_{\mathrm{s}}$ bands, over a $\sim 4$ year time period. We also noted a reddening in the $\left(H-K_{\mathrm{s}}\right)$ color as the object gets fainter in both bands. However, our previous results were based on a single epoch of NIR observations, and the large photometric uncertainties made it difficult to reliably confirm TMR-1C as a variable source.

In our present NIR imaging campaign of the TMR-1 system conducted between October 2011 and January 2012 we have obtained 44 epochs of observations in each of the $H$ and $K_{\mathrm{s}}$ filters, resulting in higher quality photometry with uncertainties a factor of $\sim 10$ smaller than previous measurements. Our present campaign covers shorter timescales of $\sim 10$ min to $\sim 4$ months, making it possible to probe short-term variability for the TMR-1 system. Also, a comparison with the previous data sets provides a $\sim 9$ year baseline to measure the proper motion and determine Taurus membership for the TMR-1 components. Section 2 provides details of the observing campaign, an analysis of the photometric and astrometric data is described in Sect. 3, results on short- and long-term NIR variability are given in Sect. 4, while Sect. 5 discusses the possible origins of variability in TMR-1C.

\section{Observations}

Our NIR imaging campaign was conducted between October 2011 and January 2012. Observations were obtained at the CFHT $3.6 \mathrm{~m}$ telescope, using the wide-field infrared camera, WIRCam. This imager covers a $20^{\prime} \times 20^{\prime}$ field of view, with a pixel scale of $0.3^{\prime \prime} /$ pix. We obtained alternate $H$ and $K_{\mathrm{s}}$ observations to check for short-term variability in each filter. In both bands, a five-point dithering pattern (20" dithering offset) was used, with five exposures obtained at each dither position. The exposure time was set to $15 \mathrm{~s}$ in the $H$ band, and $20 \mathrm{~s}$ in the $K_{\mathrm{s}}$ band. Combining the various overheads, a $\sim 14$ min observing time was required to complete one set of observations (total 25 frames) in each filter. Thus, we were able to obtain four epochs of observations per filter in two continuous hours of observing time during one night. This sequence allowed us to probe short-term variability over a timescale of few minutes to an hour. To probe variability over a week-long and a month-long period, the two-hour sequence was repeated every night for seven consecutive nights during October 2011 and then four times during the $2011 \mathrm{~A}$ semester. In this way, we were able to obtain a total of 88 epochs, i.e., 44 epochs per filter, over the full observing campaign. The shortest time difference between two sets of observations in a single filter is $\sim 14 \mathrm{~min}$, and the longest is $\sim 4$ months. In January 2012, four nights were affected by a partial cloud cover, with poor seeing of $0.85^{\prime \prime}-1.1^{\prime \prime}$. The rest of the nights were photometric, with stable seeing between $0.5^{\prime \prime}$ and $0.8^{\prime \prime}$.

The raw images were pre-processed by the WIRCam observer team using the 'I'iwi data reduction pipeline (version 2.1.1). The detrended images obtained from the pipeline first have all detector imprints removed, followed by dark subtraction, flat-fielding, and sky-subtraction. The crosstalk is removed from the final pre-processed images, and astrometry performed with an rms scatter of the resulting WCS solution between $0.3^{\prime \prime}$ and $0.8^{\prime \prime}$. To obtain a good signal-to-noise ratio we stacked the 25 images taken over $\sim 10 \mathrm{~min}$ in one set of observations for each filter. The object TMR-1C is clearly detected in the stacked images in both bands. We could not resolve the protostar TMR-1AB into its individual components, and all photometric measurements are for the composite source. The images shown in Figs. 1 and 3 are from a deep stack constructed by combining all 44 stacks.

\section{Data analysis}

\subsection{Differential photometry}

The first step in our analysis was to conduct differential photometry, and compare the rms values for the targets (TMR-1 AB and $C$ ) with the mean rms derived for a reference star sample. We selected 30 reference stars in an area of $4^{\prime} \times 4^{\prime}$ roughly centered at TMR-1AB (Fig. 1). Aperture photometry was performed for the reference and target stars in each of the stacked images using the photometry tasks under the IRAF digiphot package. We chose four different aperture radii between 1 and 10 pixels, and a sky annulus with an inner radius of 3 pixels larger than the aperture radius, and a width of 4 pixels. For the components $\mathrm{AB}$ and $\mathrm{C}$, as well as the two other objects that lie close to the nebulous region around $\mathrm{AB}$ (marked as $\mathrm{E}$ and $\mathrm{F}$ in Fig. 1), we used a different method for sky subtraction, as discussed further in Sect. 3.2. The photometry was calibrated using 2MASS magnitudes for bright sources in the same field for every epoch. The radial profiles for these bright sources were checked for any saturation effects. For each reference star, a differential magnitude was calculated by subtracting the weighted mean magnitude of all of the other reference stars from the calibrated magnitude, in each stacked image. We did various checks on the stability of the mean magnitude of the reference star sample. In each epoch, we have 30 weighted mean magnitudes. The scatter in those mean magnitudes is between $\sim 0.05$ and 0.065 mag. We were able to reduce this scatter to less than 0.05 mag by deleting one particular epoch (or one particular stack) which had bad image quality (high extinction as noted in the observing log). We also deleted the reference stars ID1, ID11, and ID24, which may be marginally variable in the $H$ or the $K_{\mathrm{s}}$ band (Sect. 4.1). We note that deleting these reference stars does not significantly reduce the rms, and the difference is found to be of $\sim 0.004$ mag in the resulting standard deviation of the mean magnitude. Another way to reduce the scatter and obtain a stable reference sample is to delete the eight faintest stars, all of which have calibrated 


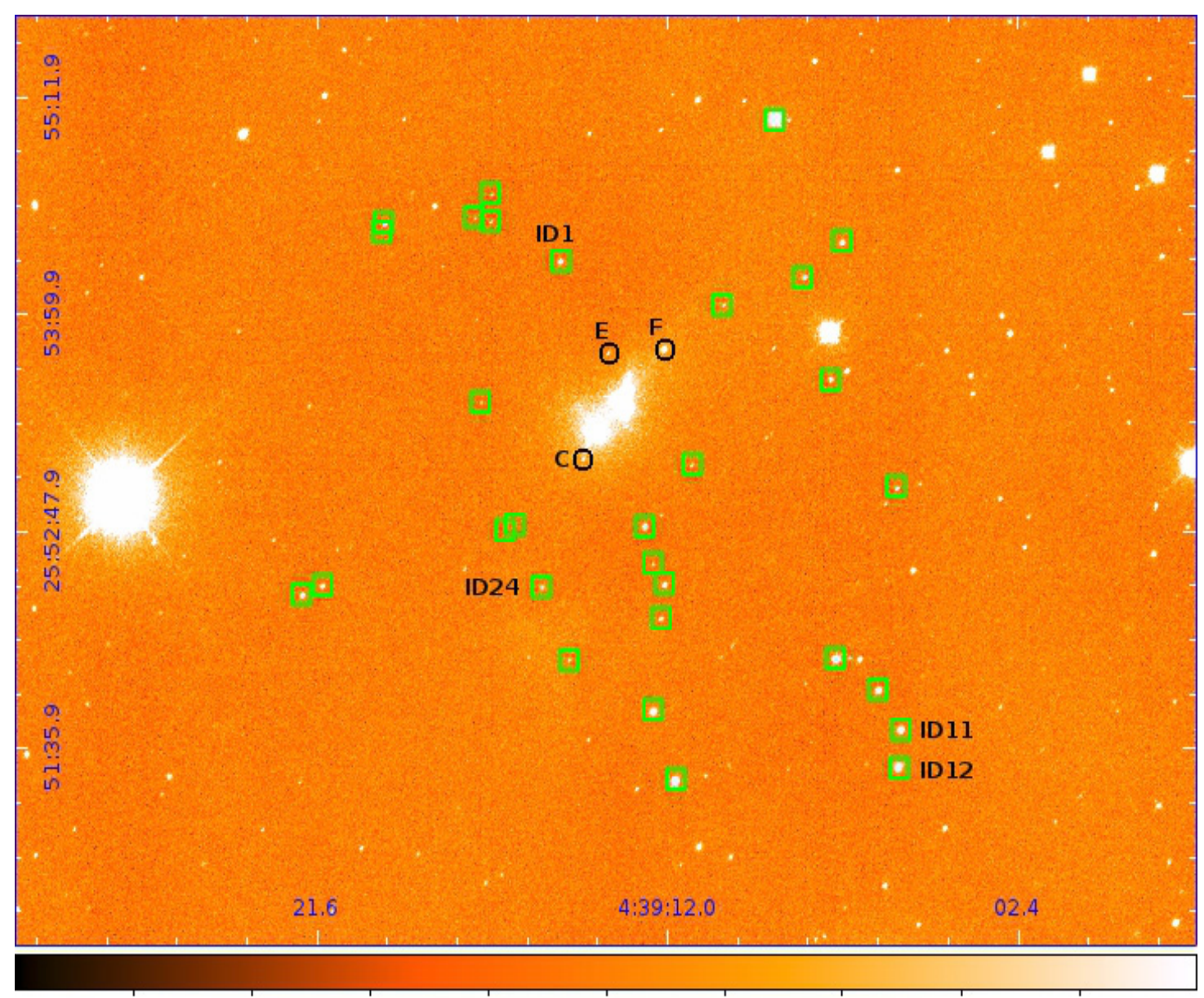

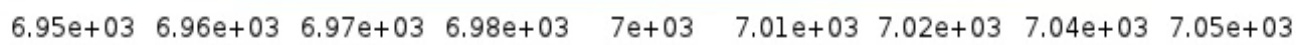

Fig. 1. WIRCam $2011 \mathrm{H}$-band false color image (stack of all 44 epochs), with the reference star sample marked as open boxes. Also denoted are some sources which show long-term variability. North is up, east is to the left. The scale bar at the bottom is in units of $\mathrm{MJy} \mathrm{sr}^{-1}$.

magnitudes of $>18$ mag; the photometry errors are also larger, $>0.07 \mathrm{mag}$. However, rejecting these sources will significantly reduce the size of a comparative sample at these faint magnitudes that we require to check for the variability of $\mathrm{C}$. These faint reference stars were thus not excluded from the sample. We applied the zero-point offset separately for each epoch, which should also result in a stable reference sample.

We then calculated the mean and the standard deviation of the distribution of differential magnitudes for each reference star from all stacked images. For AB and C, the differential magnitudes were calculated by subtracting the mean calibrated magnitude for all 30 stars in the reference sample. This method was repeated for all four aperture radii. We then plotted the mean differential magnitude versus the standard deviation for the reference star sample, and used a polynomial function to fit the distribution of points. Figure 2a shows the fits in the $H$ and $K_{\mathrm{s}}$ band for the different aperture radii used. Our reference sample consists of stars with a range in magnitude between $\sim 12$ and $18 \mathrm{mag}$ in the $H$ band, and between $\sim 11.3$ and $18.2 \mathrm{mag}$ in the $K_{\mathrm{s}}$ band. The sigma values and thus the fits in Fig. $2 \mathrm{a}$ are expected to rise towards fainter objects, i.e., for more positive values for the differential magnitude. We see this rise for aperture radii of 3 pixel or larger in the $H$ band, and 5 pixel or larger in the $K_{\mathrm{s}}$ band, whereas for smaller apertures the fits are nearly flat. The 3 pixel aperture gives the best compromise across the full range of magnitudes in the $H$ band, whereas this is found to be the case for the 5 pixel aperture in the $K_{\mathrm{s}}$ band. Therefore, the final photometry considered for the reference sample and the targets is that obtained using a 3 pixel and a 5 pixel aperture radius in the $H$ and $K_{\mathrm{s}}$ band, respectively. The appropriate aperture correction was determined from the curve of growth of the brightest star in the reference sample, and added to the calibrated photometry. The presence or absence of variability based on the differential photometry plots is discussed in Sect. 4.1.

\subsection{Photometry for $T M R-1 A B$ and $C$}

The photometry for the components $\mathrm{AB}$ and $\mathrm{C}$ can be expected to have some contamination from the surrounding nebulosity. We therefore employed a different method for sky subtraction for these components in addition to using a typical sky annulus of a certain width. We selected various sky locations around these objects, and then measured the photometry using the sky value from each of the sky locations (Fig. 3, top panel). The sky background was measured using the same aperture radius as used for the target objects. The various magnitudes obtained using this method were then compared to the photometric measurement obtained at the location of the target, using a sky annulus of 3 pixels larger than the aperture radius and a width of 4 pixels. For $\mathrm{AB}$, a grid of sky locations was selected such that there were points both inside and outside the nebulous region (Fig. 3). The photometry for $\mathrm{AB}$ is slightly brighter when 

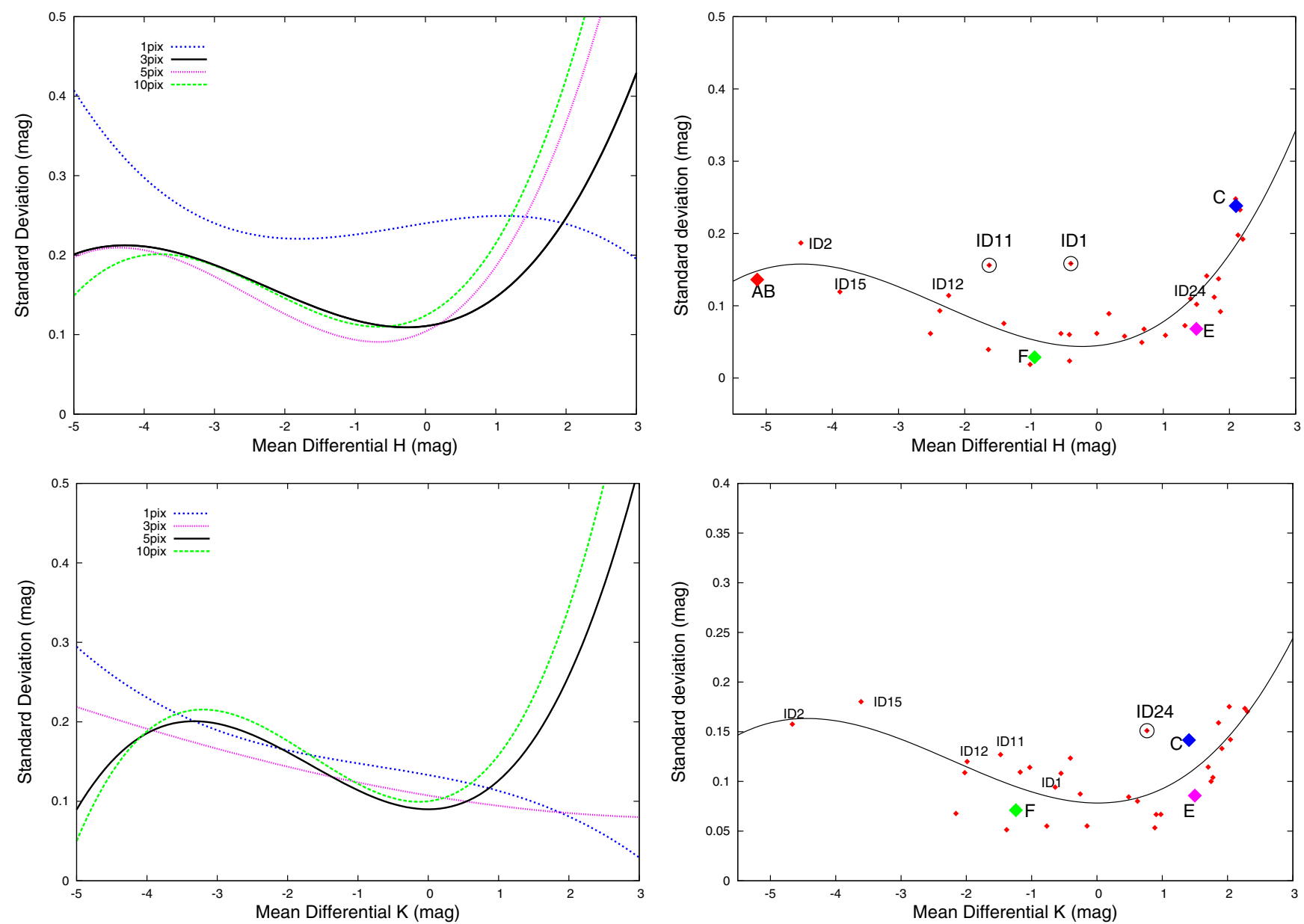

Fig. 2. Left panel: a) fitting function to the standard deviation versus the mean differential magnitude for the reference star sample, using aperture radius of $1,3,5$, and 10 pixel. The top panel shows the fits for the $H$ band, bottom panel for the $K_{\mathrm{s}}$ band. The black line represents the aperture radius considered for the photometry in the two bands. Right panel: b) standard deviation versus the mean differential magnitude for the reference star sample and the targets in the $H$ band (top) and the $K_{\mathrm{s}}$ band (bottom). Candidate variables are marked with black circles.

subtracting sky values from a less nebulous region, compared to the magnitude at the source location. The full range in magnitudes for $\mathrm{AB}$ from all sky locations is only $\sim 0.02 \mathrm{mag}$, which suggests that the surrounding nebulosity is evenly and relatively smoothly distributed around the source at a level of a few pixels. We can roughly estimate the nebulosity surface brightness per pixel by this full range in magnitude divided by the area in pixels considered for the measurements. This is found to be $\sim 0.0008 \mathrm{mag} / \mathrm{pixel}$, which is a negligible value. The main contribution to the photometry for $\mathrm{AB}$ is thus from the protostar itself, and the surrounding nebulosity does not prevent a precise measurement of the photometry. We therefore considered the photometric measurement for $\mathrm{AB}$ obtained at the source location, using a sky annulus of $30-40$ pixels. We note that $A B$ appears extended and shows a saturated radial profile for all single images in the $K_{\mathrm{s}}$ band, because of which we do not have a photometric measurement in this band. The final calibrated and aperture corrected $H$-band photometry for $\mathrm{AB}$, obtained by averaging the photometry from all 44 epochs, is listed in Table 1.

The contamination is comparatively more enhanced for the much fainter target TMR-1C. For the component $\mathrm{C}$, we selected various sky locations that lie close to and farther away from the nebulous region surrounding $\mathrm{AB}$, as marked in Fig. 3 (bottom left panel). Since the nebulous emission is stronger at locations $1-6$, the magnitudes for $\mathrm{C}$ obtained using sky background measurements at these locations is $\sim 1.7 \mathrm{mag}$ fainter than the magnitude obtained at the source location. On the other hand, at sky locations surrounding C (locations 7-10), or the ones located farther away from the nebulous region (locations 11-14), the magnitudes are brighter by $\sim 0.4$ mag than the magnitude at the source location. We can then estimate that the contamination due to the nebulous region at the location of $\mathrm{C}$ and its immediate surroundings is about $0.4 \mathrm{mag}$. To correct for the possible contamination effects, we took the mean of the sky measurements from the locations of 7-13, and then used this sky value to measure the photometry for $\mathrm{C}$. We note that if we average all of the sky locations, the resulting magnitude is $\sim 0.06$ mag fainter than averaging the 7-13 locations, which is a difference comparable to the photometric uncertainty. The difference between the two measurements is small because the low sky background at locations south or close to $\mathrm{C}$ cancel the effect of the comparatively higher sky values close to the nebulous region. Table 1 lists the final calibrated and aperture corrected photometry for $\mathrm{C}$ obtained by averaging the photometry from all 44 epochs in both bands.

We also redid the photometry for $\mathrm{AB}$ and $\mathrm{C}$ from the 2002 and 2009 CFHT observations, presented in Riaz \& Martín (2011), using the same analysis methods as employed for the 2011 data. The new photometric measurements are comparable within the uncertainties with the ones reported in our previous work. The uncertainties are a factor of $\sim 10$ smaller for the 2011 data compared to 2002 or 2009 , which can be expected considering that the present measurements have been obtained from 

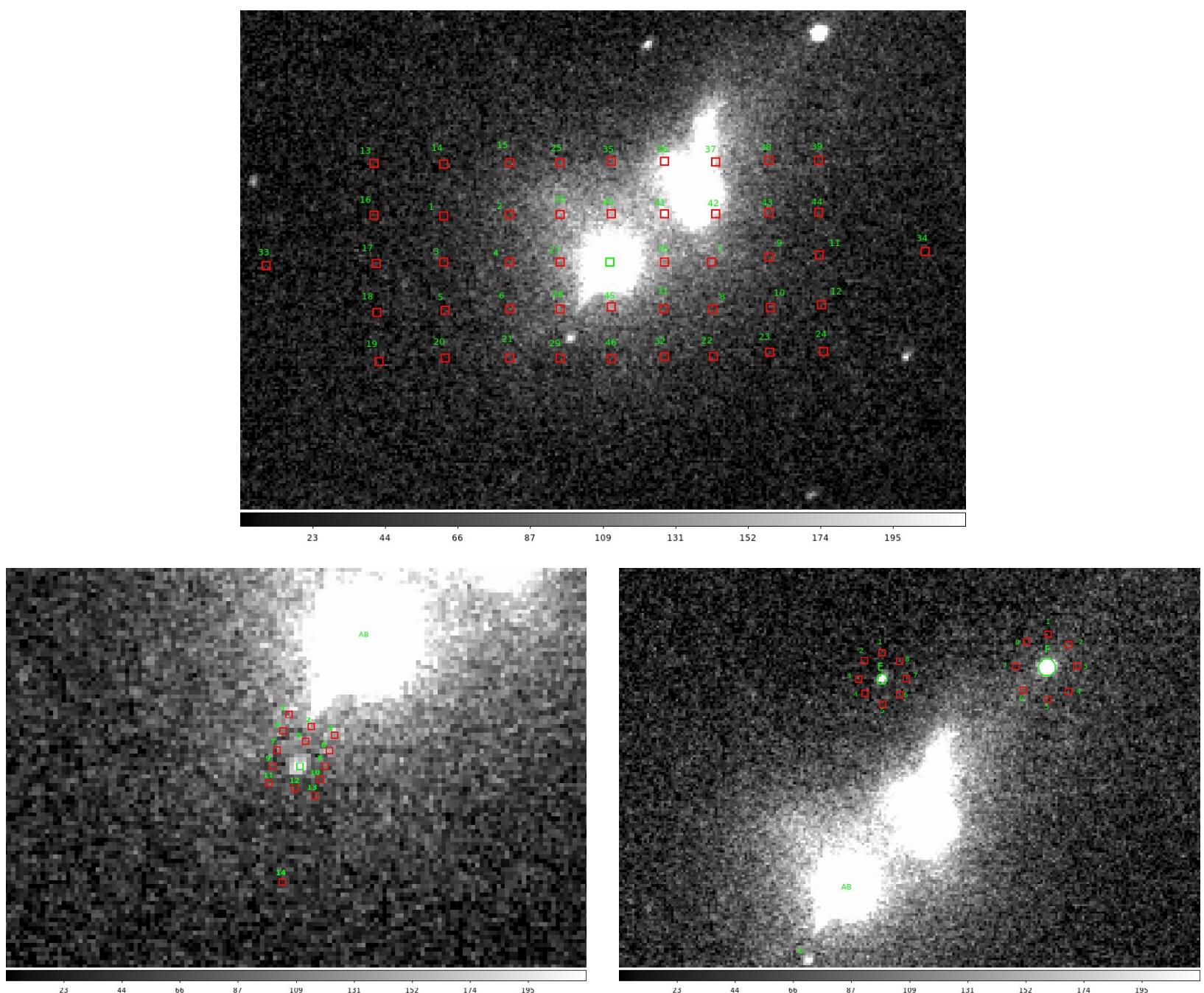

Fig. 3. Top: a) grid of sky locations (red squares) selected both inside and outside the nebulous region to measure the photometry for TMR-1AB (green square). Bottom left: b) sky locations close to and farther away from the nebulous region surrounding AB, selected to measure the photometry for $\mathrm{C}$ (green square). Bottom right: c) sky locations used to measure the photometry for $\mathrm{E}$ and $\mathrm{F}$, which lie north of TMR-1AB. These image cuts are from the stack of all 44 epochs of WIRCam $2011 \mathrm{H}$-band images; north is up, east is to the left. The scale bar at the bottom of each image is in units of $\mathrm{MJy} \mathrm{sr}^{-1}$.

nearly 1000 images in each filter. The present photometry for TMR-1AB and $\mathrm{C}$ is thus of a much higher quality and is a vast improvement over all previously reported photometric measurements for these sources, including the T98 HST observations.

Persson et al. (1998) developed a grid of $J_{-}, H_{-}, K$-, and $K_{\mathrm{s}}$-band standards for the HST/NICMOS camera, using observations from the Las Campanas Observatory (LCO) in Chile. Later, Carpenter (2001) derived 2MASS-LCO transformation equations using 2MASS photometry for 82 stars from Persson et al. (1998). Equations (1) and (2) below are the color-transformation relations from Persson et al. (1998) and Carpenter (2001), respectively.

$$
\begin{aligned}
(H-K)_{\mathrm{CIT}}=(0.974 \pm 0.020)(H-K)_{\mathrm{LCO}} \pm(0.013) \\
\left(H-K_{\mathrm{S}}\right)_{2 \mathrm{MASS}}=(1.019 \pm 0.010)(H-K)_{\mathrm{LCO}} \\
+(0.005 \pm 0.005)
\end{aligned}
$$

In Tables 1 and 3, we have listed our CFHTIR and WIRCam 2MASS-calibrated $\left(H-K_{\mathrm{s}}\right)$ color-transformed to $\left(H-K_{\mathrm{s}}\right)_{\mathrm{LCO}}$, using Eq. (1). The results are similar using Eq. (2). We find a difference in the $\left(H-K_{\mathrm{S}}\right)$ color of $\sim 0.01 \mathrm{mag}$ for TMR-1C, and 0.02-0.03 mag for TMR-1AB.

\subsection{Objects in the vicinity of $T M R-1 A B$}

In Fig. 3 (bottom, right panel), we have marked two objects, $\mathrm{E}$ and $\mathrm{F}$, which lie close to the nebulous region around $\mathrm{AB}$. The coordinates for these objects are listed in Table 4. The object $\mathrm{F}$ is a 2MASS source with the designation 2MASS J04391199+2553490. There is no spectral classification available for this object. It shows significant variability in the $(H-$ $K_{\mathrm{S}}$ ) color when comparing the 2MASS and our WIRCam or CFHTIR photometry (Table 3), as discussed further in Sect. 4.2. The object $\mathrm{E}$ has no matches in 2MASS or in SIMBAD. This is a new detection, and as discussed in Sect. 3.4, it is a strong candidate for Taurus membership based on its proper motion. For both of these objects, we used the same photometry method as employed for $\mathrm{AB}$ and $\mathrm{C}$. The sky locations are shown in Fig. 3. For E, there is a $\sim 0.3 \mathrm{mag}$ difference between the photometry at the source location and that obtained using sky measurements at locations 5-8, i.e., closer to the nebulous region. For F, there is a much smaller difference of $\sim 0.06 \mathrm{mag}$ between the magnitude at the source location and that obtained using sky measurement at any of the 1-8 locations. The final photometry for these two sources 


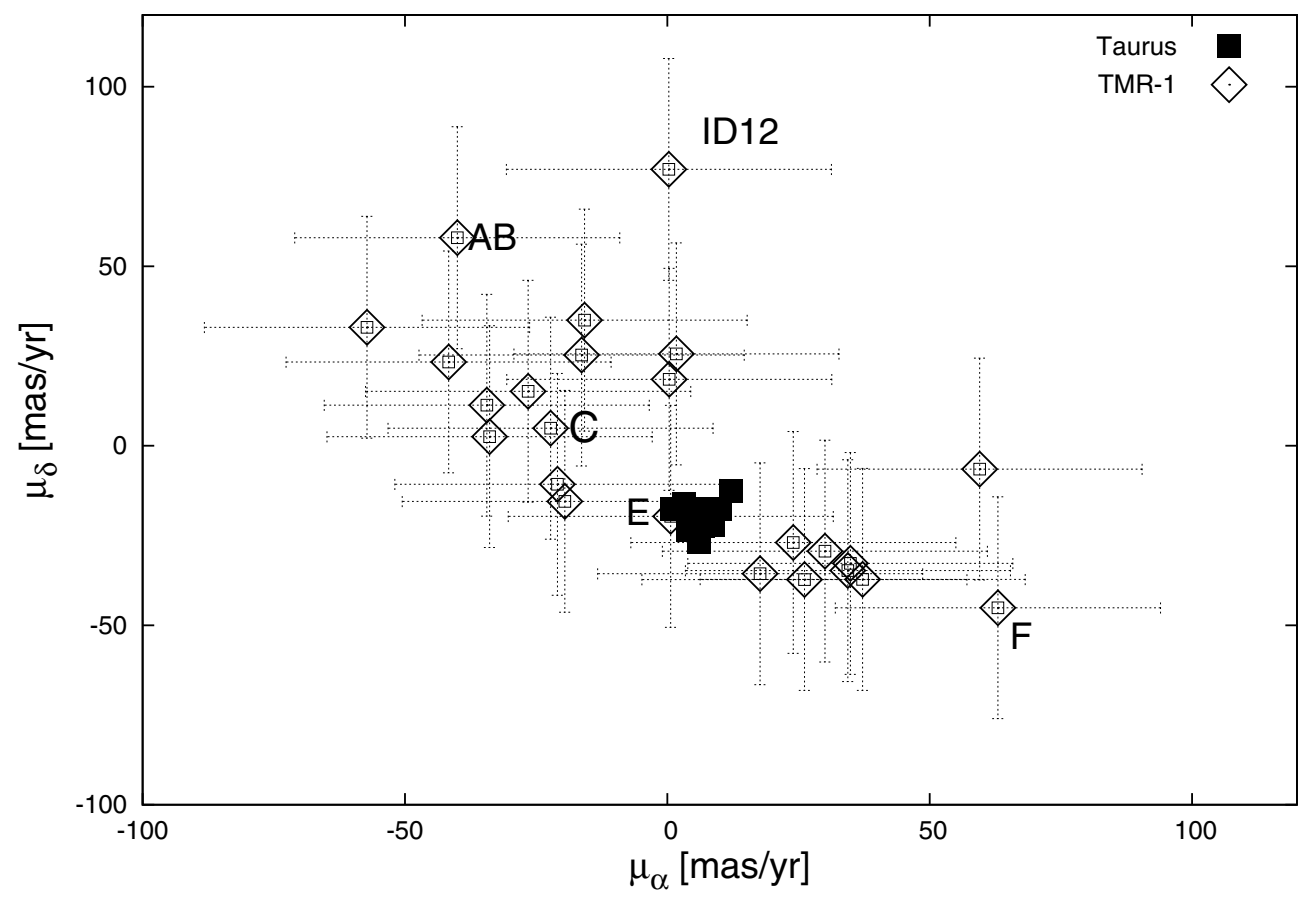

Fig. 4. Proper motion in Dec versus the proper motion in RA. Proper motions were obtained from the 2002 and 2011 $H$-band positions. Black squares denote the median proper motions for the 11 groups in Taurus from Luhman \& Mamajek (2009). The errors on proper motions for these groups are between 1 and 2 mas/yr. was obtained using the mean sky value from all sky locations (Table 3).

We cannot confirm the detection at a $>2-\sigma$ level for the faint object TMR-1D, reported in Petr-Gotzens et al. (2010). This source lies to the northwest of TMR-1AB, near the edge of a filamentary structure extending northwest from the protostar. The object TMR-1D is $\sim 1$ mag fainter than TMR-1C in the $H$ and $K_{\mathrm{s}}$ bands (Petr-Gotzens et al. 2010). It is undetected in our 2002 and 2009 observations. In the present data, a faint blob is seen in $6 K_{\mathrm{s}}$-band images from January 2012 at the TMR-1D location marked in Petr-Gotzens et al. (2010). However, the detection significance is very low and the radial profile of the blob shows only scattered emission. Deeper observations are required to conduct photometry and astrometry for this source.

\subsection{Astrometry}

The 2002 and 2011 data provide a $\sim 9$ year baseline over which the proper motions for the targets can be measured. Figure 4 plots the proper motion in RA and Dec for the reference star sample and the targets, as determined using the $H$-band observations from 2002 and 2011. Since AB is saturated in the $K_{\mathrm{s}}$ band, we preferred to use the $H$-band data for astrometric measurements. The uncertainty of $\sim 30$ mas/yr on the proper motions was calculated from the standard deviation of the full distribution of proper motions for the reference star sample. Also included for comparison in Fig. 4 are the median proper motions for the 11 groups in the Taurus-Auriga star-forming region discussed in Luhman \& Mamajek (2009). The errors on proper motions for these groups are between 1 and 2 mas/yr. The typical proper motion for Taurus from Ducourant et al. (2005) is $+2,-22 \mathrm{mas} / \mathrm{yr}$, with uncertainties of 2-5 mas/yr.

An interesting case is object $\mathrm{E}$, which has a proper motion of $+0.66,-19.6$ mas/yr and overlaps with the Taurus loci (Fig. 4), making it a strong candidate for Taurus membership. Based on its faint magnitudes (Table 3), this source is probably a brown dwarf. However, for the rest of the objects including the TMR-1 targets, the large uncertainty of $\sim 30 \mathrm{mas} / \mathrm{yr}$ on the proper motions from our 2002/2011 measurements makes it difficult to confirm Taurus membership. Figure 4 also shows the possible presence of two groups of stars based on the proper motions: group A with $\mu_{\alpha}>0$ or more positive than the Taurus loci, and group B with comparatively negative $\mu_{\alpha}$ of $\leq 0$, similar to $\mathrm{AB}$ and $\mathrm{C}$. We checked if this could be a systematic effect due to the respective position of an object on the detector, but no particular correlation is observed between the XY position and the proper motion of the sources. This subgrouping may be real, but a confirmation of it would require higher precision astrometric measurements obtained over a larger baseline than the $\sim 9$ years considered here.

Within the large uncertainties, the proper motion for TMR-1AB and $\mathrm{C}$ are comparable. The protostar $\mathrm{AB}$, however, shows a higher proper motion in declination compared to $\mathrm{C}$ (Table 3.4). The separation between $\mathrm{AB}$ and $\mathrm{C}$, as measured in all of the $H$-band stacked images, is between $9.8^{\prime \prime}$ and 11.3". The mean value is $10.2^{\prime \prime}$, with a standard deviation of $0.3^{\prime \prime}$. This is consistent with the $10^{\prime \prime}$ separation reported by Terebey et al. (1998), and the 9.8" measurement from our 2002 observations. The objects TMR-1AB and C most likely form a common proper motion pair. With a larger baseline of 20 years or more, the astrometric error bars can be expected to be much smaller than the present estimates, and the association of $\mathrm{AB}$ and $\mathrm{C}$ can then be confidently confirmed.

\section{Results}

\subsection{Short-term NIR variability}

In order to probe variability over the short-term periods of a few minutes to $\sim 4$ months probed in the 2011 observations, we compared the rms values of the differential magnitudes (Sect. 3.1) for the targets with the reference star sample, in both bands. This is shown in Fig. 2b. The solid line in this figure is a fit to the distribution of points for the reference stars, and represents the mean rms for this sample. We considered an object to be a variable source if the observed rms was a factor of 2 higher than the mean rms for the reference sample, at a given differential magnitude. In the $H$ band, both TMR-1AB and TMR-1C have rms values 

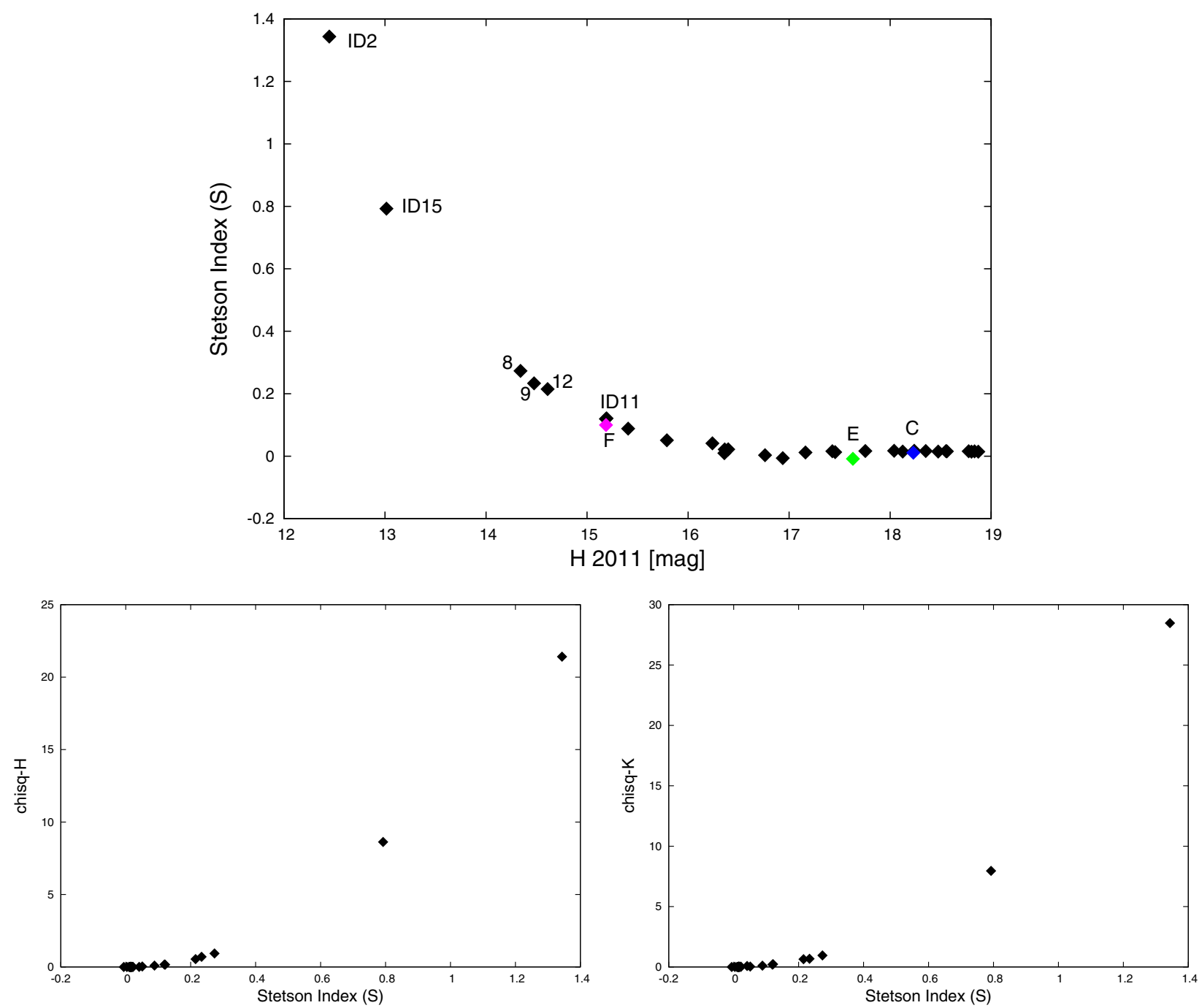

Fig. 5. Top: Stetson variability index plotted against the $H$-band (2011) magnitude. Bottom: reduced $\chi^{2}$ value of the $H$ (left) and $K_{\mathrm{s}}($ right) photometry for the reference sample, plotted against the Stetson index.

consistent with the mean for the reference sample, and thus can be considered as non-variables over this short-term period. In the $K_{\mathrm{s}}$ band, $\mathrm{C}$ lies at a $\sim 1.2$ sigma level above the mean rms curve, which is not significant enough to classify it as a variable. Objects $\mathrm{E}$ and $\mathrm{F}$ that lie close to the nebulous region also do not show any short-term variability in both bands.

We do find two references stars, ID1 and ID11, which lie at a $\sim 2$.9-sigma and $\sim 1$.9-sigma level, respectively, above the mean rms in the $H$-band differential plot (Fig. 2b). The photometry for these objects is listed in Table 3. We note that the time series plots for these two sources are similar to the other non-variable sources in the reference sample. None of these sources lie at a $\geq 2-\sigma$ level above the mean rms in the $K_{\mathrm{s}}$-band differential plot, and can be considered as non-variable in this band. ID11 shows clear signs of long-term variability over the 2002-2011 period, as discussed in Sect. 4.2.4, whereas ID1 is non-variable over this long-term period.

A well-known method of identifying variable objects is based on the Stetson variability index (Stetson 1996), which is particularly reliable when checking for correlated variations in multiband magnitudes. We calculated the Stetson variability index $S$ for each star from the observed $H$ and $K_{\mathrm{s}}$ magnitudes and associated photometric uncertainties, as described in Carpenter et al. (2001)

$S=\frac{\sum_{i=1}^{p} g_{i} \operatorname{sgn}\left(P_{i}\right) \sqrt{\left|P_{i}\right|}}{\sum_{i=1}^{n} g_{i}}$

where $p$ is the number of epochs of observations and $n$ is the number of measurements used to determine the mean magnitude. The parameter $P_{i}$ is the product of the normalized residuals of the observations in the two bands

$P_{i}=\frac{n}{n-1} \frac{m_{\mathrm{H}}-\bar{m}_{\mathrm{H}}}{\sigma_{m_{\mathrm{H}}}} \frac{m_{K_{\mathrm{s}}}-\bar{m}_{K_{\mathrm{s}}}}{\sigma_{m_{K_{\mathrm{s}}}}}$,

and $g_{i}$ is the weight assigned to each normalized residual. We assigned a weight of $1 / 2$ in each band, implying a total weight of 1.0 for detections in both bands.

Figure 5 shows the index $S$ plotted against the mean $H$-band magnitude for the reference stars, and C, E, and F. There is a trend of higher index for the brighter sources, also noted by Carpenter et al. (2001). This is to be expected, since the calculation of $S$ includes dividing the differential magnitudes by the photometry error, and the brighter sources have smaller errors. The variability cut-off in the Stetson index can be taken from 
the reduced $\chi^{2}$ value of the observed magnitudes in each band, which was calculated as

$\chi^{2}=\frac{1}{n-1} \sum_{i=1}^{n} \frac{\left(m_{i}-\bar{m}\right)^{2}}{\sigma_{i}^{2}}$.

A high $\chi^{2}$ value could result from variability or random noise. Plotting the $\chi^{2}$ values versus the Stetson index (Fig. 5), the variability cut-off can be considered to be $\chi^{2} \geq 1$ and $S \geq 0.2$. Carpenter et al. have considered the variability cut-off to be $\chi^{2} \geq 2$ and $S \geq 0.55$, but their sample also has a wider range in magnitudes and includes a high fraction of bright $(H<14$ mag) sources. We have used a lower index cut-off since we have a much fainter sample.

In Fig. 5 there are just five sources that lie above the cutoff considered, and the two strongest contenders for variability based on this criteria are the two brightest sources (ID2 and ID15). As mentioned, there is a potential bias in the index being higher for brighter sources, which may not be variable. Also, these two objects lie close to the mean fit in the differential plots (Fig. 2b), which would classify them as non-variable in both bands. For ID2, the peak counts are $\sim 55 \%$ of the saturation limit in both bands, while for ID15, the peak counts are just at the level of $\sim 50 \%$ of the saturation limit. The photometry for these two bright sources thus might be affected by non-linearity, which could result in a large Stetson index and high $\chi^{2}$ values.

The Stetson variability index for ID11 is 0.12 , below the threshold considered for variability (0.2), whereas it is much lower (0.02) for ID1. We note that the Stetson index is an indicator of correlated variability in multiple bands, and since ID 1 and 11 are both non-variable in the $K_{\mathrm{s}}$ band, a low index value can be expected. Based on these arguments, we consider ID11 to be a long-term variable object, while ID1 might be marginally variable over the short-term period in the $H$ band. The comparatively higher standard deviation in the $H$ band differential magnitude found for ID1 could be due to a sudden fluctuation in this band, which is not correspondingly observed in the $K_{\mathrm{s}}$ band. In the $K_{\mathrm{S}}$ band differential magnitude plot, object ID24 lies at a $\sim 1.87-\sigma$ level above the mean rms (Fig. 2b). This object is a non-variable in the $H$ band. We do not have the 2002 photometry for this source because it lies out of the field of view of the CFHTIR observations. Therefore, we cannot check for any long-term variability. The Stetson index for ID24 is 0.015 , which would classify it as a non-variable. Among the other sources in Fig. 5 that lie above index $S \geq 0.2$, ID8, 9, and 12, are all consistent with being non-variables from the differential photometry plots, and also do not show any signs of long-term variability (Fig. 6). To summarize, we do not find any prominent short-term variable sources in our present dataset.

\subsection{Long-term NIR variability between 2002 and 2011}

\subsubsection{Contrasting blue/red $\left(H-K_{\mathrm{s}}\right)$ colors for TMR-1C}

Table 1 lists the photometry for TMR-1C from all previous and current observations. We find clear signatures of long-term variability when comparing the 2011 photometry with the one from 2002 and earlier epochs (Fig. 6). The amplitude of variations is different in the two bands. The star TMR-1C became brighter in the $H$ band by $\sim 1.7$ mag between 1998 and 2002, and then fainter again by a smaller amplitude of $\sim 0.7$ mag between 2002 and 2011. The $H$-band photometry is nearly constant between 1998 and 2000. In contrast, TMR-1C became increasingly brighter in the $K_{\mathrm{s}}$ band in the $\sim 13$ year period between
1998 and 2011. The brightening is of $\sim 0.4$ mag between 1998 and 2002, and of a similar amplitude of $\sim 0.5$ mag between 2002 and 2009. It is again found to be slightly brighter by $\sim 0.1 \mathrm{mag}$ in the $K_{\mathrm{S}}$ band in 2011. Overall, TMR-1C became brighter by $\sim 1$ mag in both bands over the 1998-2011 time period.

The $\left(H-K_{\mathrm{s}}\right)$ color for TMR-1C shows large variations from a nearly constant red value of 1.3-1.6 mag between 1998 and 2000 to a much bluer color of $-0.1 \mathrm{mag}$ in 2002 , and then again a red color of $1.1 \mathrm{mag}$ in 2011 similar to that observed in the 1998-2000 period. The variations are at a $>2-\sigma$ level. The period of variability might be $\sim 2$ years, like that observed between 2000 and 2002, or shorter. The variations may also be aperiodic. As the $\left(H-K_{\mathrm{s}}\right)$ color gets bluer from 2000 to 2002, TMR-1C gets significantly brighter in the $H$ band (Table 1 ). When the color gets redder again between 2002 and 2011, TMR-1C gets fainter in the $H$ band but brighter in the $K_{\mathrm{s}}$ band. The difference in the trends observed in the $H$ - and $K_{\mathrm{s}}$-band variability suggests the presence of more than one origin for the observed variations (Sect. 5).

\subsubsection{Photometric variability for $T M R-1 A B$}

A comparison of the $H$-band photometry for TMR-1AB indicates brightening by $\sim 2$ mag between 1998 and 2002, and then the emission is nearly constant between 2002 and 2011 (Table 1). Based on radiative transfer modeling of the optical to millimeter SED for TMR-1AB, Furlan et al. (2008) concluded that the protobinary is viewed at a close-to edge-on inclination of $\sim 60-70^{\circ}$. The $H$-band variability observed between the 1998 and 2002 observations could be due to variable extinction by an edge-on disk or the ambient envelope material. It may also be the case that TMR-1AB is observed unocculted in all observations taken after 1998, which would explain the similar magnitudes in the 2002 and $2011 \mathrm{H}$-band observations. This protobinary is saturated in the $K_{\mathrm{s}}$-band in the 2002 and 2011 observations, therefore the only $\left(H-K_{\mathrm{s}}\right)$ color measurement available is from the 2MASS observations in 1998. The HST colors from Terebey et al. (1998) are for the individual components of TMR-1AB, which makes it difficult to make a direct comparison with the 2MASS measurement. Within the uncertainties, the 1998 2MASS $\left(H-K_{\mathrm{s}}\right)$ color of 2.24 is similar to the HST color of $\sim 2-3$ mag, although we would expect the composite photometry for the protobinary to be brighter than the individual components. Further observations are required to investigate if $\mathrm{AB}$ shows a similar blueing/reddening trend to that observed for $\mathrm{C}$.

\subsubsection{Photometric variability for objects $E$ and $F$}

Object $\mathrm{F}$ is a 2MASS source with a designation of 2MASS J04391199+2553490. A comparison of its 2MASS photometry with the 2002 and 2011 photometry indicates long-term variability in the $\left(H-K_{\mathrm{s}}\right)$ color of $\sim 1-1.4 \mathrm{mag}$ (Table 3$)$. Object $\mathrm{F}$ gets bluer between 1998 and 2002 as it gets brighter in the $H$ band. This is a trend similar to TMR-1C, and is suggestive of the same physical origin for these sources. This is also accompanied by slight brightening of $\sim 0.1 \mathrm{mag}$ in the $K_{\mathrm{s}}$ band. It gets red again between 2002 and 2011 by a smaller amplitude of $\sim 0.4$ mag as it gets fainter in the $H$ band. The emission in the $K_{\mathrm{s}}$ band is nearly constant during this time period. In comparison, object $\mathrm{E}$ shows smaller amplitude variation of $\sim 0.4 \mathrm{mag}$ in the $\left(H-K_{\mathrm{s}}\right)$ color between 2002 and 2011, and as the color gets redder, E gets fainter in both bands by $\sim 0.1-0.5 \mathrm{mag}$ (Table 3). As noted in Sect. 3.4, E is a strong contender for Taurus membership, whereas $\mathrm{F}$ appears 
as an outlier in the proper motion diagram shown in Fig. 4. There are WISE counterparts available for both E and F: however, the WISE mid-infrared images are strongly affected by the nebulosity surrounding TMR-1AB, and none of these sources could be resolved to obtain individual photometry. It is therefore difficult to confirm if $\mathrm{E}$ and $\mathrm{F}$ are disk sources, and if disk emission could be responsible for the observed variability.

\subsubsection{Two new variable sources}

We discovered two new sources, ID11 and ID12, which show long-term variability when comparing the 2002 and 2011 ( $H$ $K_{\mathrm{s}}$ ) colors (Fig. 6). The photometry and astrometry for these objects are listed in Tables 3 and 4, respectively. These are new detections and no matches were found in the SIMBAD database. Both objects are located in the southwest of TMR-1ABC (Fig. 1). Object ID11, in particular, shows large-amplitude variation of $\sim 2$ mag in the $\left(H-K_{\mathrm{s}}\right)$ color between 2002 and 2011, which is stronger than TMR-1AB or C. No short-term variability has been detected for this source in the 2011 data (Fig. 2b). As the $\left(H-K_{\mathrm{s}}\right)$ color for this object gets bluer, the $H$ magnitude gets brighter by $1.7 \mathrm{mag}$, while the $K_{\mathrm{s}}$ magnitude gets fainter by $\sim 0.3 \mathrm{mag}$ (Table 3 ). Object ID12, on the other hand, shows smaller amplitude variation of $\sim 0.5$ mag between 2002 and 2011. There are no 2MASS matches or spectral classifications available for these sources, however, we were able to find WISE counterparts for both objects. Objects ID11 and ID12 are isolated sources in WISE 3.4 and $4.6 \mu \mathrm{m}$ bands, with a detection of $>10-\sigma$, but are undetected in the 12 and $22 \mu \mathrm{m}$ bands. These sources have a [3.4]-[4.6] color of 0.3-0.4 mag, and are probably Class III objects, although a few Class II sources with weak excesses in these shorter wavelength WISE bands exhibit bluer [3.4]-[4.6] colors than the majority of the disk sources (e.g., Riaz et al. 2012). Longer wavelength observations are required to confirm if ID11 and ID12 are disk bearing objects, and if the possible origin of variability could be disk emission or variable extinction.

As can be seen in Fig. 4, the proper motion for ID11 is similar to TMR-1C, and is consistent with the Taurus loci within the large error bars. Object ID12, however, appears to be an outlier and may not be a candidate Taurus member. As discussed in Sect. 3.4, with a longer baseline of 20 years or more, outlier sources of this kind in the TMR-1 field might begin to separate from the Taurus loci, making it easier to determine their Taurus membership.

\section{Discussion}

The absence of short timescale variability for TMR-1C suggests that it may not be a YSO. The typical variations in the NIR observed for protostars and T Tauri stars in Taurus are found to be $\sim 0.1-0.3$ mag, with periods of a few days to a few weeks (e.g., Park \& Kenyon 2002). While we do not find any strong evidence of short-term variability upto a $\sim 4$ month period for TMR-1C, based on the final accuracy of our present observations we still cannot confirm small-amplitude variations of $<0.15-0.2 \mathrm{mag}$ in the NIR (Fig. 2). The non-variability on a short timescale could be due to the amplitude level being lower than our detection limit. Several optical and NIR variability surveys of ultracool dwarfs in young clusters (e.g., Bailer-Jones \& Mundt 2001; Carpenter et al. 2001; Joergens et al. 2003; Caballero et al. 2004) have found amplitudes as small as $0.5-1 \%$. On the other hand, these surveys have noted that the fraction of variable sources is never higher than $30-40 \%$. The fraction of variable Class I stars in Taurus is also found to be similar (Park \& Kenyon 2002). There can be YSOs which are not variable, and therefore the absence of short-term variability for TMR-1C, at any amplitude level, still does not reject this object as a candidate YSO. An important case to note is TMR-1AB itself, which does not show any variability in the $H$ band on a short-term timescale, nor on a long-term timescale (Fig. 2; Table 1). It may also be the case that the variability period for $\mathrm{C}$ is longer than $\sim 4$ months. The variable YSOs in Taurus and other young clusters have not been monitored over several years, and so long-term variations over a year or longer periods are not known for those objects. The various epochs of observations for TMR-1C compiled in Table 1 are randomly sampled measurements, and the period of variability could be anywhere from a few months to the two to four years suggested by these observations. There is also the possibility that TMR-1C is going through a quiescent phase, and this object might be a case similar to AA Tau, which shows variability on several time-scales, from very large amplitudes to quiescent periods where the flux is stable over a period of more than a week (e.g., Bouvier et al. 2003). While we still do not have enough information to draw a firm conclusion on the nature of TMR-1C, the arguments above provide a strong case for this source to be considered a potential YSO.

There is also the possibility that TMR-1C is a background star passing behind some patchy foreground material, and the cause of the long-term variations could be foreground extinction. We argue that if foreground extinction is the main cause of the observed variations, then this should result in simultaneous brightening or dimming in both bands, at amplitudes roughly following the extinction law (i.e., $A_{\mathrm{H}} / A_{K_{\mathrm{S}}} \sim 1.56$; Reike \& Lebofsky 1985). As noted in Sect. 4.2.1, while C shows both dimming and brightening in the $H$ band at different amplitudes, it became continuously brighter in the $K_{\mathrm{s}}$ band between 1998 and 2011 (Table 1). The absence of any particular correlation observed between brightness and color changes is actually consistent with some variable YSOs in Taurus (e.g., Park \& Kenyon 2002). We would also expect variable foreground extinction to occur on a larger spatial scale, rather than simply affecting object C. Our reference star sample is located roughly within $4^{\prime} \times 4^{\prime}$ of TMR-1C, but there are only two other objects in this region which show signs of long-term variability (Fig. 6), which may or may not be due to variable foreground extinction. We also note that the spectral energy distribution (SED) for TMR-1C cannot be fit using a model of an extincted background star, as shown in Petr-Gotzens et al. (2010). Any type of background star would have been detected in their ISAAC long wavelength images.

The possible origins for the long-term variability observed for TMR-1C were discussed in detail in Riaz \& Martín (2011) and Petr-Gotzens et al. (2010), and we refer the readers to the discussion in these papers. What we have reconfirmed in our present observations is the large-amplitude long-term variations in the NIR emission, which were observed between 1998 and 2002 , and that no particular correlation is observed between the brightness and the color changes. This suggests that more than one origin is responsible for the observed variability for C. For example, the drop in magnitude in the $H$ band over the $2002-$ 2011 period is $\sim 0.7 \mathrm{mag}$, which is much smaller than a $\Delta H$ of $\sim 1.7$ mag observed during the 1998-2002 period. This brightening could be due to physical variations in the inner disk structure, such as a change in the thickness of the inner edge or certain local high energy events like accretion shocks that can cause thermal instabilities and raise the dust temperature in the inner disk region. Any structural inhomogeneities in a circumstellar disk, or even variable extinction due to the ambient molecular cloud, 


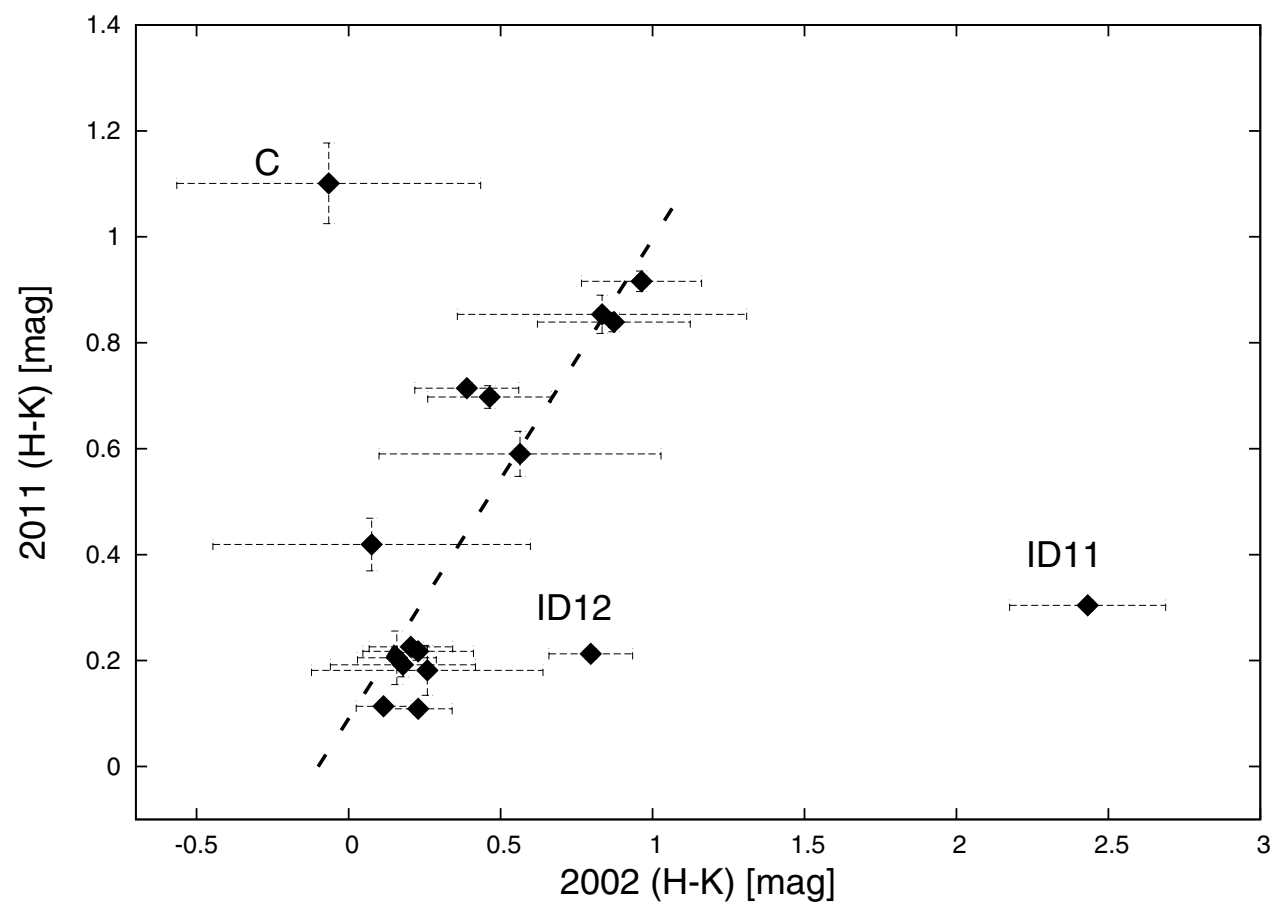

Fig. 6. 2011 versus the $2002\left(H-K_{\mathrm{s}}\right)$ color for the reference star sample and the targets. The dashed line is a linear fit to the reference star sample.

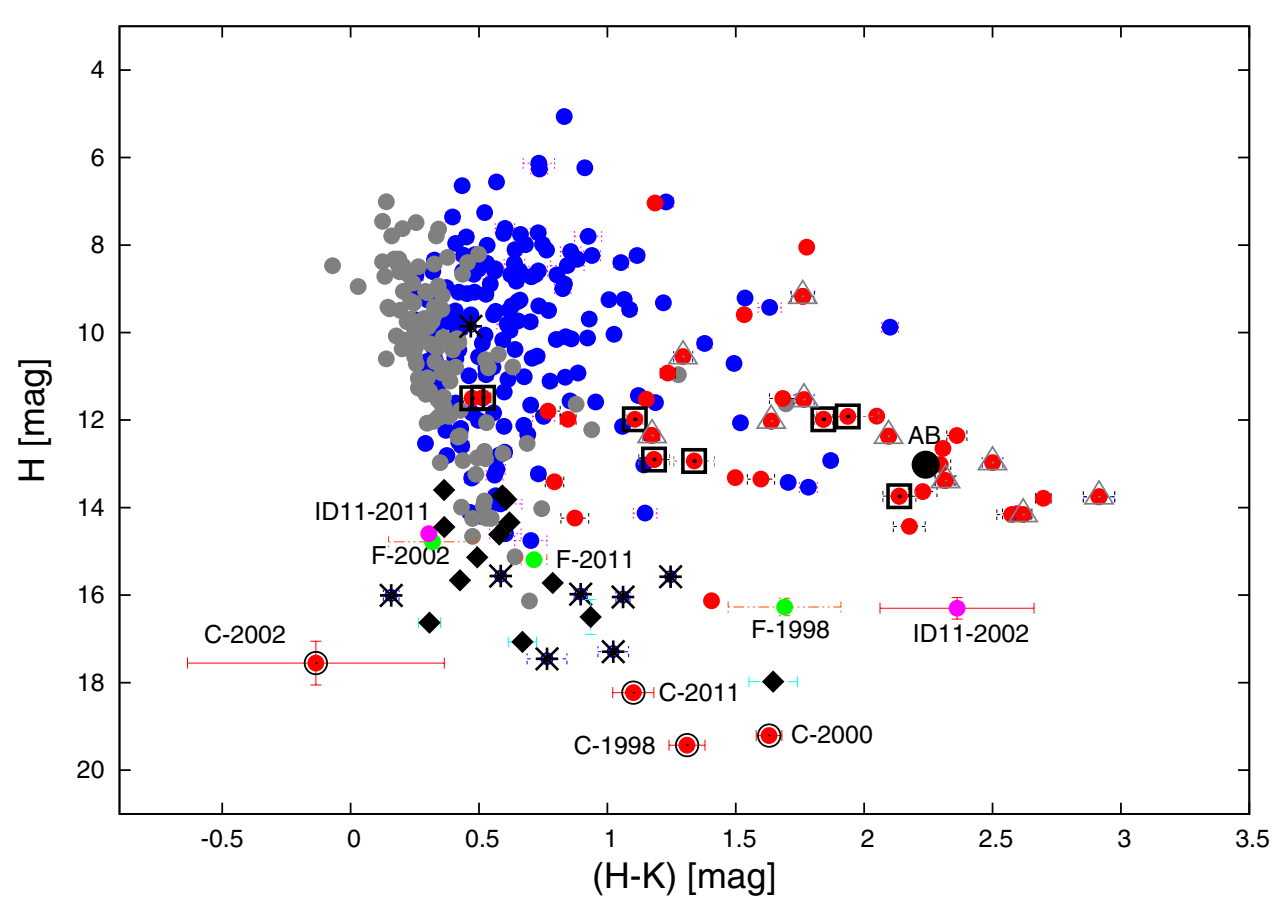

Fig. 7. NIR color-magnitude diagram comparing the TMR-1 components and other variable sources with the Class I (red), Class II (blue), and Class III (grey) sources in Taurus. The protostellar sources with face-on $\left(<50^{\circ}\right)$ and edge-on $\left(>50^{\circ}\right)$ inclinations are marked by black open squares and grey triangles, respectively. Also plotted are 2011 colors for the two groups seen in Fig. 4, based on the proper motions; asterisks represent group A with $\mu_{\alpha}>0$, filled diamonds group B with $\mu_{\alpha}<0$. can cause the object to become bluer as it gets brighter in both bands, which is found to be the case for C during the 19982002 period. We again consider the example of AA Tau, which has been suspected of being surrounded by a warped disk undergoing dynamical structural changes and causing the variability timescales to change and/or to remain quiescent over different periods of time (e.g., Bouvier et al. 2003). There could also be variations in the magnetic field strength, which can modulate the accretion flow onto the inner disk. Such complicated inner disk geometry variations are expected to create aperiodic variations, with timescales varying from a few days to years (e.g., Carpenter et al. 2001).

There is, however, no concrete evidence yet that TMR-1C is surrounded by circumstellar material. This object was undetected in the IRAC mid-infrared bands, and in the ISAAC L' data (Petr-Gotzens et al. 2010). Therefore, we cannot measure its spectral slope so as to classify it. Given the faintness of this object, the disk would be too faint to be detected at the sensitivities of the IRAC and ISAAC instruments. It is important to note that Terebey et al. (2000) and Petr-Gotzens et al. (2010) have presented low-resolution NIR spectroscopic observations of TMR-1C. Both studies have found the NIR spectrum to be featureless. The spectra do not show any prominent absorption bands due to methane or water vapor that dominate the spectra for cool objects at $T_{\text {eff }}<2000 \mathrm{~K}$. If TMR-1C is surrounded by a circumstellar disk that is at a high inclination angle to the line of sight, then the central object will be occulted by the disk, and its NIR spectrum will be dominated by the scattered light from the 
surface layers of the disk. The spectrum in this case can be expected to appear featureless. While the mid-infrared data points for TMR-1C are all upper limits, radiative transfer modeling of the near- to mid-infrared SED suggests the presence of a highly inclined disk, at an inclination angle of $87^{\circ}$ (Petr-Gotzens et al. 2010). There are several degeneracies in the model fit, but the results are consistent with the featureless NIR spectrum.

In Fig. 7, we have compared the NIR colors for TMR-1AB and $\mathrm{C}$ with other protostars and disk sources in the Taurus region. The Taurus Class I/II/III sources shown in this figure have been obtained from the work of Luhman et al. (2010). The $\left(H-K_{\mathrm{s}}\right)$ color obtained from the high-precision 1998, 2000, and 2011 photometry for TMR-1C are similar to the colors observed for the Taurus protostars, which are comparatively much brighter sources and have $\left(H-K_{\mathrm{S}}\right) \geq 1$. The object TMR-1C thus could be a very faint Class I source. Among these three measurements, there is variability in the $\left(H-K_{\mathrm{s}}\right)$ color of $\sim 0.3-$ $0.5 \mathrm{mag}$, which is similar in amplitude to the variable YSOs in Taurus. The main discrepancy for TMR-1C is the 2002 point, which is even bluer than the Class III sequence (Fig. 7). The typical intrinsic $\left(H-K_{\mathrm{s}}\right)$ color for Class I sources is estimated to be $0.6 \pm 0.4$ (e.g., Doppmann et al. 2005). As discussed in Riaz \& Martín (2011), it may be that we have measured the intrinsic colors for TMR-1C in our 2002 measurement, while the 1998, 2000, and 2011 photometry is more affected by extinction. If indeed this blue color measurement is the intrinsic color for TMR-1C, then it could even be an ultracool brown dwarf, or a $\mathrm{T}$ dwarf, which is at an early Class 0/I evolutionary stage because $\mathrm{T}$ dwarfs are known to exhibit bluer colors than late-M or L dwarfs (e.g., Marley et al. 2002). We also note that the very low bolometric luminosity of $10^{-3} L_{\odot}$ (T98) and high lineof-sight extinction of $A_{V} \sim 18 \mathrm{mag}$ (Petr-Gotzens et al. 2010) are suggestive of this object being a very low-luminosity object (VeLLO; e.g., Dunham et al. 2008), although a correct classification of a VeLLO requires an estimate on the internal luminosity of the source, without any contribution from the surrounding envelope. To summarize, TMR-1C is a strong candidate for being a YSO, the classification of which requires further investigation.

The NIR color for TMR-1AB in Fig. 7 is consistent with other protostellar sources in Taurus. As previously mentioned, TMR-1AB possesses a close to edge-on disk. For some of the known protostars in Taurus, the inclination angles have been estimated via radiative transfer modeling (e.g., Furlan et al. 2008). In Fig. 7 we have marked separately the protostars that are viewed at face-on inclinations $\left(<50^{\circ}\right.$; open triangle $)$ and edgeon inclinations $\left(>50^{\circ}\right.$; black open squares). There is no particular reddening observed in the NIR color for the edge-on sources; in fact, some of the face-on disks show redder colors than edge-on sources. This could be due to the difference in other disk/envelope parameters, such as a large cavity angle which can enhance NIR scattered emission even at intermediate inclinations, or due to different accretion properties (e.g., Riaz et al. 2009). Also plotted in Fig. 7 are the 2011 colors for the two groups of stars that were noted in Sect. 3.4 as having different sets of proper motions (Fig. 4). It appears that group A with $\mu_{\alpha}>0$ and which includes object $\mathrm{F}$, has slightly redder colors than group B, which includes object $\mathrm{E}$ and mainly follows the main-sequence or the Class III locus. The objects in group A could be low-luminosity objects without a dusty disk excess emission, while the redder group B could consist of all disk sources. The difference in the colors between the two groups is very subtle, and requires further investigation. We note that the extension of the Class III sequence towards fainter magnitudes, as mentioned above, is more highlighted if we also consider the group A and group B stars plotted in Fig. 7, although some of these faint objects could be background stars and not all are expected to be YSOs. Objects F and ID11 show trends similar to $\mathrm{C}$, with fainter magnitudes in $H$ band as the $\left(H-K_{\mathrm{s}}\right)$ color becomes redder. Both of the 2002 and 2011 color measurements for object $\mathrm{E}$ are consistent with the Class III locus (Table 3). This could be either a photospheric or a transition disk source. The redder $\left(H-K_{\mathrm{s}}\right)$ color of $\sim 0.7 \mathrm{mag}$ for ID12 suggests that this could be a Class II object. The WISE [3.4]-[4.6] colors for these sources are photospheric. Longer wavelength observations can correctly confirm if these could be transition disks with inner holes resulting in photospheric emission at near- and midinfrared wavelengths.

\section{Summary}

We have conducted a NIR photometric monitoring of the TMR-1 system, and other objects in its vicinity. Our campaign was conducted with the CFHT/WIRCam imager in the $H$ and $K_{\mathrm{s}}$ bands between October 2011 and January 2012. We do not find TMR-1AB or TMR-1C to be variable in the NIR at amplitudes of $>0.15-0.2 \mathrm{mag}$ over the short-term period of $\sim 14 \mathrm{~min}$ to $\sim 4$ months. We do find clear evidence of long-term variability for $\mathrm{C}$ when comparing the present epoch of photometry with that available from 1998, 2000, 2002, and 2009. The amplitudes of variations are different for $\mathrm{C}$ in the $H$ and $K_{\mathrm{s}}$ bands, which suggests that more than one mechanism could be the cause of the observed variability.

To summarize the nature of this object: (a) the non-detection of short-term variability is not inconsistent with TMR-1C being a YSO, considering that a large fraction of YSOs are found to be non-variable in the NIR. It may also be that the shortterm variability is at an amplitude level below our detection limit ( $0.2 \mathrm{mag}$ ), which would also be consistent with $\mathrm{C}$ being a YSO. (b) From the 1998, 2000, and 2011 photometry, which are at a much higher precision than the 2002 photometry, the $\left(H-K_{\mathrm{s}}\right)$ colors for $\mathrm{C}$ are similar to the protostars in Taurus, suggesting that it could be a faint dusty Class I source. (c) The absence of long-term variability on a large spatial scale, as well as the absence of simultaneous or correlated brightening/dimming in both $H$ and $K_{\mathrm{s}}$ bands, argue against TMR-1C being a background star affected by foreground extinction.

We have discovered a new object $(\mathrm{RA}=04: 39: 13.67$; Dec $=$ $+25: 53: 47.47$ ) with a proper motion of $+0.66,-19.66$ mas/yr (uncertainty of $\sim 30 \mathrm{mas} / \mathrm{yr}$ ), similar to the typical proper motion for Taurus (+2, $-22 \mathrm{mas} / \mathrm{yr})$, making it a strong candidate for Taurus membership. Based on its faint magnitudes $(H=17.6 \pm 0.02$; $K_{\mathrm{s}}=17.2 \pm 0.04$ ), this source is likely to be a brown dwarf. Our study has also revealed two new variable sources in the vicinity of TMR-1AB, which show long-term variations of $\sim 1-2$ mag in the NIR colors between 2002 and 2011. The proper motions measured for TMR-1AB and TMR-1C are $-40,+58$ mas/yr and $-22,+5$ mas/yr, respectively, with an uncertainty of $\sim 30 \mathrm{mas} / \mathrm{yr}$. A larger baseline of 20 years or more is required to confidently confirm the physical association of TMR-1AB and C.

Acknowledgements. E.M. was funded by Spanish ministry project AYA 201130147-C03-03. B.R. would like to thank visiting summer student C. Niven for his help with the data reduction. Based on observations obtained at the CanadaFrance-Hawaii Telescope (CFHT), which is operated by the National Research Council of Canada, the Institut National des Sciences de l'Univers of the Centre National de la Recherche Scientifique of France, and the University of Hawaii. 


\section{References}

Bailer-Jones, C. A. L., \& Mundt, R. 2001, A\&A, 367, 218

Bouvier, J., Cabrit, S., Fernandez, M., Martín, E. L., \& Matthews, J. M. 1993, A\&A, 272, 176

Bouvier, J., Grankin, K. N., Alencar, S. H. P., et al. 2003, A\&A, 409, 169

Caballero, J. A., Béjar, V. J. S., Rebolo, R., \& Zapatero Osorio, M. R. 2004, A\&A, 424, 857

Carpenter, J. M. 2001, AJ, 121, 2851

Carpenter, J. M., Hillenbrand, L. A., Skrutskie, M. F., et al. 2001, AJ, 121, 3160

Doppmann, G. W., Greene, T. P., Covey, K. R., \& Lada, C. J. 2005, AJ, 130, 1145

Ducourant, C., Teixeira, R., Périé, J. P., et al. 2005, A\&A, 438, 769

Furlan, E., McClure, M., Calvet, N., Hartmann, L., et al. 2008, ApJS, 176, 184

Herbst, W., Herbst, D. K., Grossman, E. J., \& Weinstein, D. 1994, AJ, 108, 1906

Joergens, V., Fernández, M., Carpenter, J. M., \& Neuhäuser, R. 2003, ApJ, 594, 971

Kenyon, S., \& Hartmann, L. 1995, ApJS, 101, 117

Luhman, K. L., \& Mamajek, E. 2009, ApJ, 716, L120
Luhman, K. L., Allen, P. R., Espaillat, C., Hartmann, L., \& Calvet, N. 2010, ApJS, 186, 111

Marley, M. S., Seager, S., Saumon, D., et al. 2002, ApJ, 568, 335

Park, S., \& Kenyon, S. 2002, AJ, 123, 3370

Persson, S. E., Murphy, D. C., Krzeminski, W., Roth, M., \& Rieke, M. J. 1998, AJ, 116, 2475

Petr-Gotzens, M. G., Cuby, J.-G., Smith, M. D., \& Sterzik, M. F. 2010, A\&A, 522, A78

Rieke, G. H., \& Lebofsky, M. J. 1985, 288, 618

Riaz, B., \& Martín E. L. 2011, A\&A, 525, A10

Riaz, B., Martín, E. L., Bouy, H., \& Tata, R. 2009, ApJ, 700, 1541

Riaz, B., Lodieu, N., Goodwin, S., Stamatellos, D., \& Thompson, M. 2012, MNRAS, 420, 2497

Stetson, P. B. 1996, PASP, 108, 851

Terebey, S., Beichman, C. A., Gautier, T. N.,\& Hester, J. J. 1990, ApJ, 362, L63 Terebey, S., van Buren, D., Padgett, D. L., Hancock, T., \& Brundage, M. 1998, ApJ, 507, L71 (T98)

Terebey, S., Van Buren, D., Matthews, K., \& Padgett, D. L. 2000, AJ, 119, 2341 
Table 1. Photometry for TMR-1AB and TMR-1C.

\begin{tabular}{|c|c|c|c|c|c|c|}
\hline $\begin{array}{l}\text { TMR-1 } \\
\text { component }\end{array}$ & Observation (Epoch) & Band/Filter & $\begin{array}{l}\text { Photometry } \\
{[\mathrm{mag}]}\end{array}$ & $\begin{array}{c}\left(H-K_{\mathrm{S}}\right)^{a} \\
{[\mathrm{mag}]}\end{array}$ & $\begin{array}{c}\Delta H \\
{[\mathrm{mag}]}\end{array}$ & $\begin{array}{c}\Delta K_{\mathrm{s}} \\
{[\mathrm{mag}]}\end{array}$ \\
\hline $\mathrm{C}$ & $\begin{array}{l}\text { WIRCam (2011 Oct.-2012 Jan.) } \\
\text { WIRCam (2011 Oct.-2012 Jan.) }\end{array}$ & $\begin{array}{l}H \\
K_{\mathrm{s}} \\
\end{array}$ & $\begin{array}{l}18.23 \pm 0.03 \\
17.13 \pm 0.07\end{array}$ & $1.10 \pm 0.08$ & $0.68 \pm 0.5^{b}$ & $-0.55 \pm 0.5^{b}$ \\
\hline $\mathrm{C}$ & WIRCam (2009 Jan.) & $K_{\mathrm{s}}$ & $17.2 \pm 0.3$ & & & \\
\hline $\mathrm{C}$ & $\begin{array}{l}\text { CFHTIR (2002 Oct.) } \\
\text { CFHTIR (2002 Oct.) }\end{array}$ & $\begin{array}{l}H \\
K_{\mathrm{s}} \\
\end{array}$ & $\begin{array}{l}17.55 \pm 0.5 \\
17.68 \pm 0.5\end{array}$ & $-0.135 \pm 0.5$ & $-1.68 \pm 0.5^{c}$ & $-0.44 \pm 0.5^{c}$ \\
\hline $\mathrm{C}$ & $\begin{array}{l}\text { ISAAC (2000 Oct. })^{d} \\
\text { ISAAC (2000 Oct.) }\end{array}$ & $\begin{array}{l}H \\
K_{\mathrm{s}}\end{array}$ & $\begin{array}{l}19.21 \pm 0.05 \\
17.53 \pm 0.05\end{array}$ & $1.63 \pm 0.05$ & $-0.22 \pm 0.05^{e}$ & $-0.6 \pm 0.07^{e}$ \\
\hline $\mathrm{C}$ & ISAAC $(1998 \text { Dec. })^{d}$ & $K_{\mathrm{s}}$ & $17.63 \pm 0.1$ & & & \\
\hline $\mathrm{C}$ & $\begin{array}{l}\text { HST/NICMOS }^{f} \text { (1998 Aug.) } \\
\text { HST/NICMOS (1998 Aug.) }\end{array}$ & $\begin{array}{c}F 160 W(1.6 \mu \mathrm{m}) \\
F 205 W(2.05 \mu \mathrm{m})\end{array}$ & $\begin{array}{c}19.43_{-0.05}^{+0.04} \\
18.12 \pm 0.06 \\
\end{array}$ & $1.31 \pm 0.07$ & & \\
\hline $\mathrm{AB}$ & WIRCam (2011 Oct.-2012 Jan.) & $H$ & $11.04 \pm 0.02$ & & $-0.01 \pm 0.3^{b}$ & \\
\hline $\mathrm{AB}$ & CFHTIR (2002 Oct.) & $H$ & $11.05 \pm 0.3$ & & $-1.97 \pm 0.3^{c}$ & \\
\hline A & $\begin{array}{l}\text { HST/NICMOS }^{f} \text { (1998 Aug.) } \\
\text { HST/NICMOS (1998 Aug.) }\end{array}$ & $\begin{array}{l}F 160 W(1.6 \mu \mathrm{m}) \\
F 205 W(2.05 \mu \mathrm{m})\end{array}$ & $\begin{array}{l}15.65_{-0.5}^{+0.8} \\
13.43_{-0.3}^{+0.4} \\
\end{array}$ & $2.22 \pm 0.7$ & & \\
\hline B & $\begin{array}{l}\text { HST/NICMOS }^{f} \text { (1998 Aug.) } \\
\text { HST/NICMOS (1998 Aug.) }\end{array}$ & $\begin{array}{c}F 160 W(1.6 \mu \mathrm{m}) \\
F 205 W(2.05 \mu \mathrm{m})\end{array}$ & $\begin{array}{c}17.22 \pm 1.1 \\
13.78_{-0.4}^{+1.1} \\
\end{array}$ & $3.44 \pm 1.0$ & & \\
\hline $\mathrm{AB}$ & $\begin{array}{l}\text { 2MASS (1998) } \\
\text { 2MASS (1998) }\end{array}$ & $\begin{array}{l}H \\
K_{\mathrm{s}} \\
\end{array}$ & $\begin{array}{l}13.02 \pm 0.03 \\
10.72 \pm 0.02\end{array}$ & $2.24 \pm 0.03$ & & \\
\hline
\end{tabular}

Notes. ${ }^{(a)}$ Using the color transformation relation from Persson et al. (1998); Eq. (1). ${ }^{(b)}$ The difference of the 2011 and $2002 H$ - and $K_{\mathrm{s}}$-band photometry. ${ }^{(c)}$ The difference of the 2002 and $1998 H$ - and $K_{\mathrm{s}}$-band photometry. ${ }^{(d)}$ From Petr-Gotzens et al. $(2010) .{ }^{(e)}$ The difference of the 2000 and $1998 \mathrm{H}$ - and $K_{\mathrm{s}}$-band photometry. ${ }^{(f)} \mathrm{HST} / \mathrm{NICMOS}$ photometry from T98.

Table 2. Astrometry for TMR-1AB and TMR-1C.

\begin{tabular}{|c|c|c|c|c|c|c|c|}
\hline \multirow{2}{*}{$\begin{array}{l}\text { TMR-1 } \\
\text { component }\end{array}$} & \multirow[t]{2}{*}{ Observation (Epoch) } & \multicolumn{2}{|c|}{ Position } & \multirow{2}{*}{$\begin{array}{c}\operatorname{Sep}^{a} \\
{\left[{ }^{\prime \prime}\right]}\end{array}$} & \multirow{2}{*}{$\begin{array}{c}\text { Position error } \\
{\left[{ }^{\prime \prime}\right]}\end{array}$} & \multirow{2}{*}{$\begin{array}{c}\mu_{\alpha}{ }^{b} \\
{[\mathrm{mas} / \mathrm{yr}]}\end{array}$} & \multirow{2}{*}{$\begin{array}{c}\mu_{\delta} \\
{[\mathrm{mas} / \mathrm{yr}]}\end{array}$} \\
\hline & & RA (J2000) & $\operatorname{Dec}(\mathrm{J} 2000)$ & & & & \\
\hline $\mathrm{C}$ & WIRCam (2011 Oct.-2012 Jan.) & 043914.33 & +255312.58 & 10.2 & $0.3-0.8$ & -22.243 & +4.887 \\
\hline $\mathrm{AB}$ & WIRCam (2011 Oct.-2012 Jan.) & 043913.98 & +255322.12 & & $0.3-0.8$ & -40.027 & +57.95 \\
\hline $\mathrm{C}$ & WIRCam (2009 Jan.) & 043914.22 & +255311.33 & & $0.3-0.8$ & & \\
\hline $\mathrm{C}$ & CFHTIR (2002 Oct.) & 043914.22 & +255312.18 & 9.8 & 0.1 & & \\
\hline $\mathrm{AB}$ & CFHTIR (2002 Oct.) & 043913.88 & +255321.20 & & 0.1 & & \\
\hline $\mathrm{C}$ & HST/NICMOS ${ }^{c}$ (1998 Aug.) & 043914.14 & +255311.8 & 10 & 0.35 & & \\
\hline A & HST/NICMOS (1998 Aug.) & 043913.84 & +25 5320.6 & & 0.35 & & \\
\hline $\mathrm{B}$ & HST/NICMOS (1998 Aug.) & 043913.83 & +255320.4 & & 0.35 & & \\
\hline $\mathrm{AB}$ & 2MASS (1998) & 043913.89 & +255320.88 & & 0.08 & & \\
\hline
\end{tabular}

Notes. ${ }^{(a)}$ Separation ["] between TMR-1AB and TMR-1C. ${ }^{(b)}$ Proper motion obtained from the 2002 and $2011 H$-band positions. Errors on $\mu_{\alpha}$ and $\mu_{\delta}$ are $30.97 \mathrm{mas} / \mathrm{yr}$ and $30.90 \mathrm{mas} / \mathrm{yr}$, respectively. ${ }^{\left({ }^{c}\right)}$ HST/NICMOS positions from T98. 
Table 3. Photometry for objects E, F, and variable candidates.

\begin{tabular}{|c|c|c|c|c|}
\hline Object & Observation (Epoch) & Band/Filter & $\begin{array}{l}\text { Photometry } \\
{[\mathrm{mag}]}\end{array}$ & $\begin{array}{c}\left(H-K_{\mathrm{s}}\right) \\
{[\mathrm{mag}]}\end{array}$ \\
\hline $\mathrm{E}$ & $\begin{array}{l}\text { WIRCam (2011 Oct.-2012 Jan.) } \\
\text { WIRCam (2011 Oct.-2012 Jan.) }\end{array}$ & $\begin{array}{l}H \\
K_{\mathrm{s}}\end{array}$ & $\begin{array}{c}17.632 \pm 0.023 \\
17.2127 \pm 0.044\end{array}$ & $0.41 \pm 0.05$ \\
\hline $\mathrm{E}$ & $\begin{array}{l}\text { CFHTIR (2002 Oct.) } \\
\text { CFHTIR (2002 Oct.) } \\
\end{array}$ & $\begin{array}{l}H \\
K_{\mathrm{s}} \\
\end{array}$ & $\begin{array}{c}17.099 \pm 0.4 \\
17.093 \pm 0.336 \\
\end{array}$ & $0.006 \pm 0.5$ \\
\hline $\mathrm{F}$ & $\begin{array}{l}\text { WIRCam (2011 Oct.-2012 Jan.) } \\
\text { WIRCam (2011 Oct.-2012 Jan.) }\end{array}$ & $\begin{array}{l}H \\
K_{\mathrm{s}}\end{array}$ & $\begin{array}{c}15.188 \pm 0.003 \\
14.4736 \pm 0.004\end{array}$ & $0.7144 \pm 0.005$ \\
\hline $\mathrm{F}$ & $\begin{array}{l}\text { CFHTIR (2002 Oct.) } \\
\text { CFHTIR (2002 Oct.) }\end{array}$ & $\begin{array}{l}H \\
K_{\mathrm{s}}\end{array}$ & $\begin{array}{c}14.7824 \pm 0.14 \\
14.4635 \pm 0.098\end{array}$ & $0.3189 \pm 0.17$ \\
\hline $\mathrm{F}$ & $\begin{array}{l}\text { 2MASS (1998) } \\
\text { 2MASS (1998) } \\
\end{array}$ & $\begin{array}{l}H \\
K_{\mathrm{s}}\end{array}$ & $\begin{array}{c}16.27 \pm 0.20 \\
14.58 \pm 0.1 \\
\end{array}$ & $1.69 \pm 0.22$ \\
\hline ID1 & $\begin{array}{l}\text { WIRCam (2011 Oct.-2012 Jan.) } \\
\text { WIRCam (2011 Oct.-2012 Jan.) }\end{array}$ & $\begin{array}{l}H \\
K_{\mathrm{s}}\end{array}$ & $\begin{array}{l}15.7972 \pm 0.017 \\
15.0995 \pm 0.013\end{array}$ & $0.6977 \pm 0.02$ \\
\hline ID1 & $\begin{array}{l}\text { CFHTIR (2002 Oct.) } \\
\text { CFHTIR (2002 Oct.) } \\
\end{array}$ & $\begin{array}{l}H \\
K_{\mathrm{s}} \\
\end{array}$ & $\begin{array}{c}15.3994 \pm 0.16 \\
15.0055 \pm 0.126 \\
\end{array}$ & $0.3939 \pm 0.2$ \\
\hline ID2 & $\begin{array}{l}\text { WIRCam (2011 Oct.-2012 Jan.) } \\
\text { WIRCam (2011 Oct.-2012 Jan.) }\end{array}$ & $\begin{array}{l}H \\
K_{\mathrm{s}}\end{array}$ & $\begin{array}{c}16.8282 \pm 0.037 \\
16.6467 \pm 0.03\end{array}$ & $0.1815 \pm 0.05$ \\
\hline ID2 & $\begin{array}{l}\text { CFHTIR (2002 Oct.) } \\
\text { CFHTIR (2002 Oct.) } \\
\end{array}$ & $\begin{array}{l}H \\
K_{\mathrm{s}} \\
\end{array}$ & $\begin{array}{c}16.6394 \pm 0.3 \\
16.4505 \pm 0.25 \\
\end{array}$ & $0.1889 \pm 0.38$ \\
\hline ID11 & $\begin{array}{l}\text { WIRCam (2011 Oct.-2012 Jan.) } \\
\text { WIRCam (2011 Oct.-2012 Jan.) }\end{array}$ & $\begin{array}{l}H \\
K_{\mathrm{s}}\end{array}$ & $\begin{array}{c}14.593 \pm 0.004 \\
14.2889 \pm 0.007\end{array}$ & $0.3042 \pm 0.008$ \\
\hline ID11 & $\begin{array}{l}\text { CFHTIR (2002 Oct.) } \\
\text { CFHTIR (2002 Oct.) } \\
\end{array}$ & $\begin{array}{l}H \\
K_{\mathrm{s}} \\
\end{array}$ & $\begin{array}{c}16.3014 \pm 0.245 \\
13.9395 \pm 0.1 \\
\end{array}$ & $2.3619 \pm 0.3$ \\
\hline ID12 & $\begin{array}{l}\text { WIRCam (2011 Oct.-2012 Jan.) } \\
\text { WIRCam (2011 Oct.-2012 Jan.) }\end{array}$ & $\begin{array}{l}H \\
K_{\mathrm{s}}\end{array}$ & $\begin{array}{l}14.0096 \pm 0.004 \\
13.7969 \pm 0.005\end{array}$ & $0.2127 \pm 0.006$ \\
\hline ID12 & $\begin{array}{l}\text { CFHTIR (2002 Oct.) } \\
\text { CFHTIR (2002 Oct.) } \\
\end{array}$ & $\begin{array}{l}H \\
K_{\mathrm{s}} \\
\end{array}$ & $\begin{array}{c}14.6684 \pm 0.11 \\
13.9415 \pm 0.1 \\
\end{array}$ & $0.7269 \pm 0.13$ \\
\hline ID15 & $\begin{array}{l}\text { WIRCam (2011 Oct.-2012 Jan.) } \\
\text { WIRCam (2011 Oct.-2012 Jan.) }\end{array}$ & $\begin{array}{l}H \\
K_{\mathrm{s}} \\
\end{array}$ & $\begin{array}{l}12.4135 \pm 0.03 \\
12.1606 \pm 0.03\end{array}$ & $0.253 \pm 0.04$ \\
\hline ID24 & $\begin{array}{l}\text { WIRCam (2011 Oct.-2012 Jan.) } \\
\text { WIRCam (2011 Oct.-2012 Jan.) }\end{array}$ & $\begin{array}{l}H \\
K_{\mathrm{s}}\end{array}$ & $\begin{array}{l}17.5247 \pm 0.03 \\
16.3925 \pm 0.04 \\
\end{array}$ & $1.1322 \pm 0.05$ \\
\hline
\end{tabular}

Table 4. Astrometry for objects E, F, ID11, and ID12.

\begin{tabular}{cccccc}
\hline \hline Object & Observation (Epoch) & \multicolumn{2}{c}{ Position } & $\mu_{\alpha}{ }^{a}$ & $\mu_{\delta}{ }{ }^{2}$ \\
& & RA (J2000) & Dec (J2000) & {$[\mathrm{mas} / \mathrm{yr}]$} & {$[\mathrm{mas} / \mathrm{yr}]$} \\
\hline E & WIRCam (2011 Oct.-2012 Jan.) & 043913.6735 & +255347.476 & +0.6599 & -19.665 \\
$\mathrm{E}$ & CFHTIR (2002 Oct.) & 043913.5465 & +255347.328 & & \\
\hline F & WIRCam (2011 Oct.-2012 Jan.) & 043912.1618 & +255348.820 & +63.005 & -45.11699 \\
F & CFHTIR (2002 Oct.) & 043911.9933 & +255348.928 & & \\
F & 2MASS (1998) & 043911.990 & +255349.06 & & \\
\hline ID11 & WIRCam (2011 Oct.-2012 Jan.) & 043905.648 & +255142.586 & -26.52699 & +15.18299 \\
ID11 & CFHTIR (2002 Oct.) & 043905.5393 & +255142.090 & & \\
\hline ID12 & WIRCam (2011 Oct.-2012 Jan.) & 043905.7054 & +255130.399 & +0.29299 & +76.995 \\
ID12 & CFHTIR (2002 Oct.) & 043905.5787 & +255129.285 & & \\
\hline
\end{tabular}

Notes. ${ }^{(a)}$ Proper motion obtained from the 2002 and $2011 \mathrm{H}$-band positions. Errors on $\mu_{\alpha}$ and $\mu_{\delta}$ are 30.97 mas/yr and 30.90 mas/yr, respectively. 